\title{
Propriedades tecnológicas de painéis cimento-madeira produzidos com partículas de eucalipto
}

\author{
Technological properties of cement-wood panels produced with eucalypt particles
}

\section{Setsuo Iwakiri ${ }^{1}$, Rosilani Trianoski ${ }^{1}$, Alexsandro Bayestorff da Cunha ${ }^{2}$, José Guilherme Prata ${ }^{1}$, Massayuki Hara $^{3}$, Narciso Fernando Bila ${ }^{1}$, Reinaldo Calçada Guina Luis ${ }^{4}$ e Roberto Daniel Araújo ${ }^{4}$}

Recebido em 16/04/2015 / Aceito em 03/08/2015

\section{RESUMO}

O estudo teve como objetivo determinar as propriedades tecnológicas de painéis cimentomadeira produzidos com partículas de Eucalyptus benthamii Maiden et Cambage. O plano experimental foi formado por quatro composições com diferentes tratamentos das partículas (água fria, água quente, hidróxido de sódio $(\mathrm{NaOH})$ e hidróxido de cálcio $\left(\mathrm{Ca}(\mathrm{OH})_{2}\right)$, e uma composição testemunha. Os painéis foram produzidos com massa específica nominal de $1,20 \mathrm{~g} / \mathrm{cm}^{3}$, com relação madeira:cimento de 1:2,75 e água:cimento de 0,40 . Os painéis foram avaliados quanto as suas propriedades físicas e mecânicas por meio da norma europeia EN. Foram realizadas análises para verificar a ocorrência de outliers, normalidade dos dados e homogeneidade das variâncias, sendo posteriormente submetidas à análise da variância e teste de Tukey. Os resultados das propriedades físicas e mecânicas dos painéis indicaram que o melhor método de tratamento de partículas foi com o uso de $\mathrm{Ca}(\mathrm{OH})_{2}$. Com exceção do módulo de ruptura, o inchamento em espessura, módulo de elasticidade e tração perpendicular, atenderam às especificações de painéis comerciais produzidos pelo processo BISON. Dessa forma, verificou-se a viabilidade de uso da madeira de Eucalyptus benthamii para produção de painéis cimento-madeira.

PALAVRAS-CHAVE: Eucalyptus benthamii, produto para a construção civil, partículas de madeira, aglutinante mineral.

\section{ABSTRACT}

The study aimed to determine the technological properties of cement-wood panels produced with the particle Eucalyptus benthamii Maiden et Cambage. The experiment plan consisted of four compositions with different treatments of the particles (cold water, hot water, sodium hidroxyde $(\mathrm{NaOH})$ and calcium hidroxyde $\left(\mathrm{Ca}(\mathrm{OH})_{2}\right)$, and a control composition. The panels were produced with nominal specific gravity of $1.20 \mathrm{~g} \mathrm{~cm}^{-3}$, with the ratios wood:concrete $1: 2.75$ and water:cement 0.40 . The panels were evaluated for their physical and mechanical properties through the European standard EN. Analyses were performed to verify the occurrence of outliers, data normality and homogeneity of variances, and subsequently submitted to analysis of variance and Tukey test. The results of physical and mechanical properties of the panels indicated that the best method of treating particles is through the use of $\mathrm{Ca}(\mathrm{OH})_{2}$. With the exception of the modulus of rupture, the thickness swelling, modulus of elasticity and tensile perpendicular, they met the specifications of commercial panels produced by the BISON process. Therefore, the feasibility of use of Eucalyptus benthamii for wood-cement panels production was discovered.

KEYWORDS: Eucalyptus benthamii, product for the building industry, wood particles, mineral binder.

\footnotetext{
${ }^{1}$ Universidade Federal do Paraná, Curitiba, PR, Brasil.

${ }^{2}$ Universidade do Estado de Santa Catarina, Lages, SC, Brasil.

${ }^{3}$ Universidade Tecnológica Federal do Paraná, Curitiba, PR, Brasil.

${ }^{4}$ Instituto Nacional de Pesquisas da Amazônia, Manaus, AM, Brasil.

*Autor para correspondência <setsuo@ufpr.br>.
} 


\section{INTRODUÇÃO}

Os painéis cimento-madeira são produtos amplamente empregados na construção civil, principalmente em países asiáticos e europeus, como material de fechamento de paredes externas. É um produto similar aos painéis aglomerados, com diferenças relacionadas ao tipo de aglutinante e processo de prensagem, que nesse caso, utiliza cimento e prensagem à temperatura ambiente.

Os painéis cimento-madeira apresentam diversas vantagens em relação a outros materiais na construção civil, tais como: são produtos virtualmente incombustíveis; apresentam alta resistência mecânica e à umidade; são resistentes ao ataque de agentes biodegradadores; e são isolantes térmicos e acústicos (MOSLEMI 1974).

Os painéis cimento-madeira ainda não são produzidos comercialmente no Brasil, entretanto, apresentam grande potencial para a sua introdução, tendo em vista a necessidade do setor de construção civil por materiais alternativos de baixo custo para viabilizar a produção de habitações em larga escala. Outros fatores que podem facilitar a manufatura do produto no Brasil é a disponibilidade da matéria-prima na forma de resíduos do processamento mecânico da madeira e a autossuficiência do país na produção do cimento Portland.

Entre os parâmetros que influenciam na produção dos painéis cimento-madeira, destaca-se a espécie, principalmente no que se refere à composição química da madeira. Segundo SIMATUPANG et al. (1978), os extrativos presentes na madeira são os principais responsáveis pela inibição da cura e solidificação do cimento, sendo seus princípios ativos os compostos fenólicos e os carboidratos livres. Os autores afirmam também que as espécies de folhosas tropicais apresentam influência negativa mais pronunciada na pega e endurecimento do cimento do que as de coníferas. BERALDO et al. (2002) citam que a presença de açucares e extrativos solúveis em água ou álcali retarda o tempo de pega do cimento. Para viabilizar o uso de determinadas espécies com alto teor de extrativos inibidores de cura do cimento, MOSLEMI et al. (1983) recomendam três métodos de tratamentos de partículas: imersão em água fria por 24 horas; imersão em água quente por seis horas; e imersão em solução de $\mathrm{NaOH}$ (1\%) por duas horas.

Além da espécie, outros parâmetros importantes no processo de produção de painéis cimento- madeira são a relação madeira:cimento, a relação água:cimento, o tipo e quantidade de acelerador de cura. LATORRACA \& IWAKIRI (2005) afirmam que a relação madeira:cimento mais empregada industrialmente é de 1:2,75. SIMATUPANG et al. (1978) relatam que, para manufatura de painéis cimento-madeira de alta massa específica, é utilizada uma proporção de água em relação ao cimento de 0,4 . Os aditivos químicos são empregados com propósito de reduzir o tempo de endurecimento do cimento, acelerando a pega e cura. Os sais como cloreto de cálcio, cloreto de magnésio, silicato de sódio e silicato de potássio, são alguns dos aditivos utilizados, sendo que o mais empregado é o cloreto de cálcio. MOSLEMI et al. (1983) e RASHWAN et al. (1992) recomendam como quantidades ideais para uso no processo produtivo, proporções de 3 a $5 \%$ de cloreto de cálcio em relação ao peso do cimento.

O presente estudo teve como objetivo determinar as propriedades tecnológicas de painéis cimento-madeira produzidos com partículas de Eucalyptus benthamii Maiden et Cambage, com intuito de apresentar ao setor da construção civil brasileiro um produto comercial com uma espécie florestal alternativa.

\section{MATERIAL E MÉTODOS}

A madeira utilizada no presente estudo foi proveniente de um plantio experimental de $E$. benthamii com 13 anos de idade, pertencente à empresa Klabin S.A., localizado no município de Palmeira, Santa Catarina. Como aglutinante mineral foi utilizado o cimento Portland e como acelerador de cura o cloreto de cálcio.

As toras foram desdobradas tangencialmente na empresa Madepar Indústria e Comércio de Madeiras Ltda. (Lages, Santa Catarina), originando tábuas com uma polegada de espessura, as quais foram seccionadas em blocos com dimensões compatíveis com o gerador de partículas. Nesta etapa, a espessura foi reduzida para $0,7 \mathrm{~mm}$. Após a pré-secagem ao teor de umidade entre 15 a $20 \%$, as partículas foram reprocessadas no moinho de martelo para redução nas dimensões. Após a drenagem da água e lavagem para remoção de extrativos da superfície das partículas, realizou-se a secagem ao teor de umidade médio de $12 \%$ e o peneiramento para retirada dos "finos".

O plano experimental, apresentado na Tabela 1, foi composto por quatro tratamentos, que envolveram 
Tabela 1 - Plano experimental.

Table 1 - Experimental plan.

\begin{tabular}{cc}
\hline Tratamento & Pré-tratamento \\
\hline 1 & Testemunha \\
2 & Imersão 24 horas em água fria \\
3 & Imersão seis horas em água quente $\left(60{ }^{\circ} \mathrm{C}\right)$ \\
4 & Imersão duas horas em solução diluída $(1 \%)$ de $\mathrm{NaOH}$ \\
5 & $\mathrm{Ca}(\mathrm{OH})_{2}$ \\
\hline
\end{tabular}

diferentes métodos de tratamentos das partículas e tratamento testemunha.

$\mathrm{Na}$ formação dos painéis, procedeu-se inicialmente o cálculo de cada um dos componentes da mistura: partículas de madeira, cimento, água e cloreto de cálcio. Na definição da quantidade de cada componente da mistura foram consideradas as seguintes proporções: relação madeira:cimento de 1:2,75; fator água:cimento de 0,4 e $3 \%$ de cloreto de cálcio em relação ao peso do cimento. Os cálculos foram realizados para massa específica nominal do painel de 1,20 $\mathrm{g} \mathrm{cm}^{-3}$. Devidamente pesados, os componentes foram misturados numa betoneira para obtenção de massa homogênea.

A massa de cada painel, composta pelas quatro matérias-primas, foi pesada e distribuída aleatoriamente na caixa formadora do colchão, com dimensões de $385 \mathrm{~mm}$ de largura e $505 \mathrm{~mm}$ de comprimento. Sobre o colchão, foi colocada uma placa de alumínio untada com óleo mineral para facilitar o desmolde após prensagem. Após a retirada da caixa formadora, foi colocada outra placa na parte superior do colchão e duas barras de ferro de $15 \mathrm{~mm}$ para controle da espessura do painel.

O colchão foi prensado à temperatura ambiente com pressão específica de $40 \mathrm{kgf} \mathrm{cm}^{-2}$, com três painéis sobrepostos em sequência e mantidos sob restrição no aparato de grampeamento por período de 24 horas para o endurecimento. Após esse período, os grampos foram retirados e os painéis foram acondicionados na câmara climática à temperatura de $20 \pm 2{ }^{\circ} \mathrm{C}$ e umidade relativa de $65 \pm 5 \%$, por um período de 28 dias para maturação final.

Para avaliação das propriedades físicas e mecânicas, foram retirados de cada painel, três corpos-de-prova para ensaios de absorção de água e inchamento em espessura após 2 e 24 horas de imersão em água, cinco corpos-de-prova para tração perpendicular (ligação interna) e quatro corpos-deprova para flexão estática (módulo de elasticidade MOE e módulo de ruptura - MOR). Os ensaios foram realizados de acordo com os procedimentos descritos nas normas EN 317 (2002), EN 319 (2002) e EN 310 (2002), respectivamente.

Os dados obtidos nos ensaios foram submetidos ao teste de Grubss para avaliar a ocorrência de outliers; teste de Shapiro Wilks para avaliação da normalidade de dados, Bartlett para verificação da homogeneidade de variâncias, Análise de Variância e comparação de médias de Tukey. Os resultados das propriedades mecânicas foram submetidos à análise de covariância, ajustando-se os valores para uma massa específica aparente $(12 \%)$ de $1,27 \mathrm{~g} / \mathrm{cm}^{3}$. Todos os testes foram efetuados a partir do pacote estatístico STATGRAPHICS CENTURION XVI (2009), a 95\% de probabilidade.

\section{RESULTADOS E DISCUSSÃO}

\section{Propriedades físicas dos painéis}

$\mathrm{Na}$ Tabela 2, estão apresentados os valores médios de massa específica aparente dos painéis obtidos para os tratamentos analisados.

$\mathrm{Na}$ massa específica aparente dos painéis, que influencia diretamente nas demais propriedades, observa-se que a amplitude entre os valores médios encontrados foi de $1,19 \mathrm{~g} \mathrm{~cm}^{-3}$ a $1,36 \mathrm{~g} \mathrm{~cm}^{-3}$, com baixos coeficientes de variação dentro de cada um dos tratamentos, o que demonstra homogeneidade da produção dentro de cada situação. Assim, a diferença estatística encontrada entre os tratamentos e em torno da massa específica nominal pode ser atribuída às condições experimentais (IWAKIRI et al. 2012), onde ocorrem perdas de materiais durante as etapas do processo de formação dos painéis, retorno em espessura após a prensagem e a dispersão da massa 
para uma área maior que a planejada do painel (ELEOTÉRIO 2000).

$\mathrm{Na}$ Tabela 3, estão apresentados os resultados de absorção de água e inchamento em espessura após duas e 24 horas de imersão em água.

Os valores médios de absorção de água após duas horas variaram de 6,61\% (T5) a 9,00\% (T4). Os painéis produzidos com partículas tratadas com $\mathrm{Ca}(\mathrm{OH})_{2}$ apresentaram média estatisticamente igual aos painéis produzidos com partículas tratadas em água fria e água quente, e superior aos painéis produzidos com partículas sem tratamento e tratadas com $\mathrm{NaOH}$.

Para absorção de água 24 horas, os valores médios variaram na faixa de $10,90 \%$ (T5) a $14,22 \%$ (T4). Os painéis produzidos com partículas tratadas com $\mathrm{Ca}(\mathrm{OH})_{2}$ apresentaram média estatisticamente superior em relação aos painéis de todos os demais tratamentos. Não houve diferença significativa entre os painéis produzidos com partículas sem tratamento e tratadas em água fria, água quente e $\mathrm{NaOH}$.

Os valores obtidos foram satisfatórios em relação aos resultados apresentados na literatura. IWAKIRI et al. (2012) encontraram para painéis cimento-madeira de paricá e embaúba, valores de absorção de água 24 horas na faixa de $23,22 \%$ a $25,27 \%$ e de $17,64 \%$ a $20,86 \%$, respectivamente. IWAKIRI \& PRATA (2008) encontraram para painéis cimento-madeira de Eucalyptus grandis Hill ex Maiden e Eucalyptus dunnii Maiden, valores de absorção de água 24 horas na faixa de $15,15 \%$ a $20,34 \%$ e de $14,27 \%$ a $20,30 \%$, respectivamente. SILVA et al. (2005) obtiveram para painéis cimentomadeira produzidos com diferentes proporções de madeira e casca de Eucalyptus urophylla S.T. Blake, absorção de água 24 horas variando de $9,98 \%$ a $11,96 \%$.

Os valores médios de inchamento em espessura após duas horas variaram na faixa de $0,35 \%$ (T3) a $0,61 \%$ (T1). Entretanto, não foram constatadas

Tabela 2 - Resultados médios da massa específica aparente.

Table 2 - Average results of apparent density.

\begin{tabular}{ccc}
\hline Tratamento $^{\mathrm{I}}$ & Massa específica aparente $\left(\mathrm{g} \mathrm{cm}^{-3}\right)$ & $\mathrm{CV}(\%)$ \\
\hline 1 & $1,26 \mathrm{~b}$ & 5,96 \\
2 & $1,28 \mathrm{~b}$ & 5,88 \\
3 & $1,19 \mathrm{c}$ & 4,71 \\
4 & $1,27 \mathrm{~b}$ & 4,08 \\
5 & $1,36 \mathrm{a}$ & 4,83
\end{tabular}

I Tratamentos: 1 = Testemunha, 2 = Imersão 24 horas em água fria, 3 = Imersão seis horas em água quente $\left(60{ }^{\circ} \mathrm{C}\right), 4=$ Imersão duas horas em solução diluída $(1 \%)$ de $\mathrm{NaOH}$ e $5=\mathrm{Ca}(\mathrm{OH})_{2}$. Médias seguidas da mesma letra na mesma coluna são estatisticamente iguais pelo Teste de Tukey a $95 \%$ de probabilidade.

Tabela 3 - Resultados médios da absorção de água e inchamento em espessura após duas e 24 horas de imersão. Table 3 - Average results of water absorption and thickness swelling after two and 24 hours water soaking.

\begin{tabular}{cllll}
\hline Tratamento $^{\mathrm{I}}$ & AA 2h $(\%)^{\mathrm{II}}$ & AA 24h $(\%)^{\mathrm{II}}$ & IE 2h $(\%)^{\mathrm{II}}$ & ${\text { IE } 24 \mathrm{~h}(\%)^{\mathrm{II}}}^{\mathrm{II}}$ \\
\hline 1 & $8,87_{(28,89)} \mathrm{a}$ & $14,14_{(19,50)} \mathrm{a}$ & $0,61_{(38,05)} \mathrm{a}$ & $0,79_{(46,10)} \mathrm{a}$ \\
2 & $7,95_{(35,47)} \mathrm{ab}$ & $12,97_{(20,68)} \mathrm{a}$ & $0,54_{(41,62)} \mathrm{a}$ & $0,76_{(53,85)} \mathrm{a}$ \\
3 & $7,17_{(35,19)} \mathrm{ab}$ & $13,45_{(20,68)} \mathrm{a}$ & $0,35_{(48,34)} \mathrm{a}$ & $0,71_{(43,52)} \mathrm{a}$ \\
4 & $9,00_{(37,22)} \mathrm{a}$ & $14,22_{(19,96)} \mathrm{a}$ & $0,54_{(42,45)} \mathrm{a}$ & $0,92_{(40,61)} \mathrm{a}$ \\
5 & $6,6_{(25,96)} \mathrm{b}$ & $10,90_{(15,83)} \mathrm{b}$ & $0,52_{(37,92)} \mathrm{a}$ & $0,71_{(53,19)} \mathrm{a}$
\end{tabular}

${ }^{\mathrm{I}}$ Tratamentos: 1 = Testemunha, 2 = Imersão 24 horas em água fria, $3=$ Imersão seis horas em água quente $\left(60{ }^{\circ} \mathrm{C}\right)$, $4=$ Imersão duas horas em solução diluída $(1 \%)$ de $\mathrm{NaOH}$ e $5=\mathrm{Ca}(\mathrm{OH}){ }_{2} .{ }^{\text {II }} \mathrm{AA}$ : absorção de água; IE: inchamento em espessura; valores subescritos referem-se ao coeficiente de variação (\%). Médias seguidas da mesma letra na mesma coluna são estatisticamente iguais pelo Teste de Tukey a 95\% de probabilidade. 
diferenças estatísticas significativas entre os painéis de diferentes tratamentos.

Para inchamento em espessura 24 horas, os valores médios variaram na faixa de $0,71 \%$ (T3 e T5) a $0,92 \%$ (T4). Da mesma forma como observado para inchamento em espessura duas horas, não foram constatadas diferenças estatísticas significativas entre os painéis de diferentes tratamentos. Todos os resultados obtidos atendem às especificações de painéis comerciais produzidos pelo processo BISON (1978) para valores de inchamento em espessura na faixa de $1,2 \%$ a $1,8 \%$.

Com relação aos valores apresentados na literatura, os resultados de inchamento em espessura 24 horas obtidos para E. benthamii podem ser considerados satisfatórios. Para painéis produzidos com madeira de paricá e embaúba, IWAKIRI et al. (2012) encontraram valores de IE 24 horas na faixa de $1,38 \%$ a $1,95 \%$ e de 0,39 a $0,92 \%$, respectivamente. Nos estudos realizados com E. grandis e E. dunnii, IWAKIRI \& PRATA (2008) obtiveram valores de IE 24 horas variando de $2,26 \%$ a $4,13 \%$ e de $2,18 \%$ a $3,68 \%$, respectivamente.

\section{Propriedades mecânicas dos painéis}

Na Tabela 4 estão apresentados os resultados médios obtidos para os módulos de ruptura e elasticidade à flexão estática e tração perpendicular dos painéis.

Os valores médios de módulo de ruptura (MOR) variaram na faixa de 4,11 MPa (T5) a 5,77 MPa (T2). Os painéis produzidos com partículas tratadas em água fria apresentaram média estatisticamente igual aos painéis produzidos com partículas sem tratamento e tratadas com $\mathrm{NaOH}$, e superior em comparação aos painéis produzidos com partículas tratadas em água quente e $\mathrm{Ca}(\mathrm{OH})_{2}$. Os resultados obtidos ficaram abaixo das especificações de painéis comerciais produzidos pelo processo BISON (1978) para valores de módulo de ruptura mínimo de 9,0 MPa.

Para fins comparativos com resultados apresentados na literatura, IWAKIRI et al. (2012) encontraram para painéis cimento-madeira de paricá e embaúba, valores de módulo de ruptura na faixa de $6,48 \mathrm{MPa}$ a $7,91 \mathrm{MPa}$ e de $6,89 \mathrm{MPa}$ a 8,32 $\mathrm{MPa}$, respectivamente. IWAKIRI \& PRATA (2008) encontraram para painéis cimento-madeira de E. grandis e E. dunnii, valores de MOR na faixa de 6,47 $\mathrm{MPa}$ a 9,90 $\mathrm{MPa}$ e de 3,53 $\mathrm{MPa}$ a 4,02 $\mathrm{MPa}$, respectivamente. OKINO et al. (2004) encontraram para painéis cimento-madeira produzidos com madeiras de diferentes clones de Hevea brasiliensis Willd. Ex Adr, de Juss MOR variando de 4,80 MPa a 8,70 MPa.

Com relação ao módulo de elasticidade (MOE), os valores médios obtidos variaram na faixa de 2.612 MPa (T3) a $3.820 \mathrm{MPa}$ (T5). Os painéis produzidos com partículas tratadas com $\mathrm{Ca}(\mathrm{OH})_{2}$ apresentaram média estatisticamente igual aos painéis produzidos com partículas tratadas em água fria, e superior em comparação aos painéis produzidos com partículas sem tratamento e tratadas com água quente e $\mathrm{NaOH}$. Os resultados obtidos para painéis produzidos com partículas tratadas em água fria e com $\mathrm{Ca}(\mathrm{OH})_{2}$, atendem às especificações de painéis comerciais produzidos pelo processo BISON (1978) para valores de módulo de elasticidade mínimo de $3.000 \mathrm{MPa}$.

Com relação a outras pesquisas publicadas, pode-se citar SILVA et al. (2005) que encontraram para painéis cimento-madeira produzidos com diferentes proporções de mistura de madeira e casca de E. urophylla, valores de MOE entre $1.588 \mathrm{MPa}$ e $3.102 \mathrm{MPa}$; e IWAKIRI et al. (2012) que encontraram para painéis cimento-madeira de paricá e embaúba, valores de MOE entre 2.571 MPa e 3.203 MPa e de $2.836 \mathrm{MPa}$ a $3.539 \mathrm{MPa}$, respectivamente.

Para tração perpendicular, os valores médios obtidos variaram na faixa de $0,50 \mathrm{MPa}$ (T1) a $0,73 \mathrm{MPa}$ (T5). Os painéis produzidos com partículas tratadas com $\mathrm{Ca}(\mathrm{OH})_{2}$ apresentaram média estatisticamente superior aos painéis produzidos com partículas sem tratamento e tratadas em água fria, água quente e $\mathrm{NaOH}$. Todos os resultados obtidos atendem às especificações de painéis comerciais produzidos pelo processo BISON (1978) para valores de tração perpendicular mínimo de 0,40 $\mathrm{MPa}$.

Os valores obtidos foram satisfatórios em relação aos resultados apresentados na literatura. SILVA et al. (2005) obtiveram para painéis cimentomadeira produzidos com diferentes proporções de mistura de madeira e casca de E. urophylla, valores de tração perpendicular na faixa de $0,55 \mathrm{MPa}$ a 0,66 MPa. OKINO et al. (2004) encontraram para painéis cimento-madeira produzidos com madeiras de Hevea brasiliensis provenientes de diferentes clones, valores de tração perpendicular na faixa de $0,27 \mathrm{MPa}$ a 0,45 $\mathrm{MPa}$.

Os resultados das propriedades físicas e mecânicas dos painéis foram satisfatórios quando comparados com outros estudos e com as normas 
Tabela 4 - Resultados médios de módulo de ruptura, módulo de elasticidade e tração perpendicular.

Table 4 - Average results of modulus of rupture, modulus of elasticity and internal bond.

\begin{tabular}{cccc} 
Tratamento $^{\mathrm{I}}$ & MOR $(\mathrm{MPa})^{\mathrm{II}}$ & ${\text { MOE }(\mathrm{MPa})^{\mathrm{II}}}$ & ${\text { Tração perpendicular }(\mathrm{MPa})^{\mathrm{II}}}$ \\
\hline 1 & $5,27_{(24,18)} \mathrm{ab}$ & $2.854,66_{(22,12)} \mathrm{b}$ & $0,50_{(24,71)} \mathrm{b}$ \\
2 & $5,77_{(19,92)} \mathrm{a}$ & $3.243,24_{(11,10)} \mathrm{ab}$ & $0,51_{(25,10)} \mathrm{b}$ \\
3 & $4,68_{(17,53)} \mathrm{bc}$ & $2.612,85_{(14,09)} \mathrm{b}$ & $0,53_{(21,27)} \mathrm{b}$ \\
4 & $5,43_{(12,35)} \mathrm{ab}$ & $2.882,36_{(13,45)} \mathrm{b}$ & $0,53_{(12,73)} \mathrm{b}$ \\
5 & $4,11_{(19,46)} \mathrm{c}$ & $3.820,50_{(19,85)} \mathrm{a}$ & $0,73_{(19,05)} \mathrm{a}$
\end{tabular}

I Tratamentos: 1 = Testemunha, 2 = Imersão 24 horas em água fria, 3 = Imersão seis horas em água quente $\left(60{ }^{\circ} \mathrm{C}\right)$, 4 = Imersão duas horas em solução diluída $(1 \%)$ de $\mathrm{NaOH}$ e $5=\mathrm{Ca}(\mathrm{OH})_{2}{ }^{.}{ }^{\mathrm{II}} \mathrm{MOR}$ : módulo de ruptura; MOE: módulo de elasticidade; valores subescritos referem-se ao coeficiente de variação (\%); valores ajustados por ANCOVA para uma massa específica aparente de $1,27 \mathrm{~g} \mathrm{~cm}^{-3}$. Médias seguidas da mesma letra na mesma coluna são estatisticamente iguais pelo Teste de Tukey a $95 \%$ de probabilidade.

internacionais de qualidade, necessitando de ajustes somente no que tange ao comprimento das partículas, de forma a atender os valores de resistência a flexão estática (MOR). Desta forma, pode-se dizer que o $E$. benthamii, espécie alternativa plantada nas regiões frias do sul do Brasil, apresenta mais um potencial uso dentro do setor de base florestal, o que vem a contribuir para o aumento das áreas de plantio da espécie e para a introdução dos painéis cimento-madeira na região, de forma a aumentar a competitividade no mercado, principalmente na construção civil.

\section{CONCLUSÕES}

Os resultados obtidos no presente estudo permitiram concluir quanto: as propriedades físicas e mecânicas: o tratamento composto por painéis com partículas tratadas com hidróxido de cálcio $\left(\mathrm{Ca}(\mathrm{OH})_{2}\right)$ apresentou os melhores valores médios com exceção do módulo de ruptura. Assim, pode-se dizer que foi o melhor método de tratamento das partículas; a outras pesquisas publicadas: os valores médios encontrados foram satisfatórios; aos painéis comerciais produzidos pelo processo BISON: todos os valores médios encontrados para a estabilidade dimensional dos painéis e para as propriedades mecânicas atenderam as especificações, com exceção do módulo de ruptura a flexão estática.

Os resultados obtidos apontam que a madeira do Eucalyptus benthamii apresenta propriedades físicas e mecânicas satisfatórias para o uso em painéis cimento-madeira, necessitando somente de ajustes nas dimensões das partículas, de forma a atender as normas de qualidade.

\section{REFERÊNCIAS}

BERALDO AL et al. 2002. Compósito à base de resíduos vegetais e cimento portland. In: VIII Encontro Brasileiro em Madeiras e Estruturas de Madeira. Resumos... Uberlândia: EBRAMEM. p.85-90.

BISON. 1978. Wood-cement board. Berlin: BISON. 10p. (Bison Report 8).

ELEOTÉRIO JR. 2000. Propriedades físicas de painéis MDF de diferentes densidades e teores de resina. Dissertação (Mestrado em Ciências). Piracicaba: ESALQ. $121 \mathrm{p}$.

EN 310. EUROPEAN STANDARD. Determination of modulus of elasticity in bending and of bending strength. 2002.

EN 317. EUROPEAN STANDARD. Determination of swelling in thickness after immersion in water. 2002.

EN 319. EUROPEAN STANDARD. Determinação da resistência à tração perpendicular às faces da placa. 2002. IWAKIRI S et al. 2012. Avaliação do potencial de utilização da madeira de Schizolobium amazonicum "paricá" e Cecropia hololeuca "embaúba" para produção de painéis cimento-madeira. Cerne 18: 303-308.

IWAKIRI S \& PRATA JG. 2008. Utilização da madeira de Eucalyptus grandis e Eucalyptus dunnii na produção de painéis de cimento-madeira. Cerne 14: 75-81.

LATORRACA JVF \& IWAKIRI S 2005. Painéis de cimento-madeira. Painéis de madeira reconstituída. Curitiba: Fupef. p.213-239.

MOSLEMI AA. 1974. Particleboard. London: Southern Illinois University. $245 \mathrm{p}$.

MOSLEMI et al. 1983. Effect of various treatments and additives on Wood-portland cement-water systems. Wood Fiber Sci 15: 164-176.

OKINO EYA et al. 2004. Chapa aglomerada de cimentomadeira de Hevea brasiliensis Müll. Arg. Rev Árvore 28: 451-457. 
RASHWAN MS et al. 1992. Development of a lightweight low-cost concrete block using Wood residues. For Prod J 42: 57-64.

SILVA, GC et al. 2005. Produção do compósito madeira e casca de Eucalyptus urophylla S. t. Blake e cimento portland. Sci Forest 68: 59-67.

SIMATUPANG MH et al. 1978. Small scale plants for the manufacture of mineral-bonded wood composites. Resumos... Jakarta: WORLD FOR CONGRESS. p. 21.

STATGRAPHICS 2009. Statgraphics Centurion XVI. StatPoint Technologies, Inc.: Warrenton, Virgina. 